This item was submitted to Loughborough's Research Repository by the author.

Items in Figshare are protected by copyright, with all rights reserved, unless otherwise indicated.

\title{
Resonant effects in scattering by periodic arrays
}

PLEASE CITE THE PUBLISHED VERSION

LICENCE

CC BY-NC-ND 4.0

\section{REPOSITORY RECORD}

Linton, C.M., and lan Thompson. 2019. "Resonant Effects in Scattering by Periodic Arrays". figshare. https://hdl.handle.net/2134/2290. 


\title{
Resonant effects in scattering by periodic arrays
}

\author{
C. M. Linton* and I. Thompson
}

Department of Mathematical Sciences, Loughborough University, Loughborough, Leics. UK

*Email: c.m.linton@lboro.ac.uk

\begin{abstract}
The scattering of plane acoustic waves by an infinite periodic array of circles is considered. Attention is focused on parameters (frequency, incident angle, array spacing) that lead to resonance; that is, when one or more of the waves that is diffracted by the array propagates along the array. By considering the unknowns in the solution as functions of the resonant mode scattering angle, we are able to determine the precise nature of the behaviour of the solution at resonance and thereby to accurately compute the resonant state. Both single resonance, when a single mode propagates along the array, and double resonance, when there are two resonant modes propagating in opposite directions along the array, are considered. Numerical results are presented, with particular emphasis on computations of the scattered field at resonance. Comparisons are also made with scattering by a long finite array.
\end{abstract}

\section{Introduction}

Resonant phenomena caused by the interaction of plane waves and periodic arrays of scatterers have been studied for over a century. Resonances can be of different types, but perhaps the most important class are those discovered experimentally by Wood in 1902 [1]. The first theoretical treatment was due to Rayleigh [2] and since then numerous authors have studied the problem from both a theoretical and experimental point of view; a review of early work can be found in [3]. Rayleigh's treatment had limited validity, but he did establish the fundamental property of the so-called Wood's anomalies: that they occur when one of the scattered waves propagates along the array.

When a plane wave strikes an infinite periodic structure, a number of plane waves are scattered, all at different angles. The number of these modes depends on the relationship between the frequency of the incident field and the spacing between the elements of the array. Wood observed in optical diffraction gratings that the amplitude of these scattered waves can sometimes undergo a vary rapid variation when the incident field is changed only slightly and these variations were termed anomalies because there was no established theory to explain them. The process by which this happens is now well understood. It is due to the cutting on or off of a different scattered mode and can be thought of as a rapid redistribution of energy over the set of scattered modes as one passes through the cut-off point. At the cut-off point itself, one particular mode propagates along the array.

The object of this paper is to study the diffracted field at these cut-off values in a particular case using full linear diffraction theory. Specifically we treat a a two-dimensional acoustic diffraction problem where the scatterer is an infinite periodic one-dimensional array of circles. An efficient method for computing the solution in the special case when one of 
the scattered waves propagates along the array is presented. No such method appears to exist in the literature; other authors have derived only approximate results valid near to resonant states, or studied the behaviour of mode amplitudes as resonance is approached (see $[4,5,6,7]$, for example).

Aside from being of independent interest, the results obtained here are useful in several other contexts. For example, in a forthcoming study of the semi-infinite array (which is itself motivated by the desire to better understand the interaction of waves with large, finite arrays), the quantity that must be computed is the difference between the solutions to the infinite and semi-infinite problems. It is essential to be able to calculate the infinite array solution accurately at all frequencies, including at resonance. Moreover, certain functions that appear below are of importance in dealing with the excitation of an infinite array by a line source [8], and a careful treatment of that problem requires that the behaviour of these functions as resonance is approached is properly understood.

In section 2 the problem of plane wave scattering by an infinite periodic array of circles is formulated and the conditions for resonance identified. There are two cases to consider: either a single mode will be resonant, propagating one way or the other along the array, or there will be two resonant modes propagating along the array, one in either direction. The former case is treated in section 3 and the more complicated double resonance in section 4 . Numerical results are presented in section 5.

\section{General theory}

We consider a two-dimensional scattering problem which has application in a number of physical contexts. We will refer primarily to the acoustic setting in which we look for time-harmonic solutions $\operatorname{Re}[\phi(x, y) \exp (-\mathrm{i} \omega t)]$ so that the acoustic potential $\phi$ satisfies the two-dimensional Helmholtz equation

$$
\left(\nabla^{2}+k^{2}\right) \phi=0
$$

in the region exterior to the scatterers, where $k=\omega / c$ and $c$ is the speed of sound. The scatterers can be taken as either rigid (in which case the normal derivative of $\phi$ must vanish on the boundary; we call this the Neumann problem) or acoustically soft (in which case the appropriate boundary condition is $\phi=0$; we call this the Dirichlet problem). In electromagnetic theory, the boundary-value problems for the rigid and soft cases are also applicable to the scattering by an array of perfect conductors of an $S$-polarized or $P$-polarized incident field, respectively. Alternatively we can consider surface water wave scattering by vertical circular cylinders extending throughout the water depth (assumed constant). In this case, if $h$ is the water depth, with $z=0$ the undisturbed free surface and $z$ measured vertically upwards, we look for solutions of Laplace's equation of the form $\operatorname{Re}[\phi(x, y) \cosh (k(z+h)) \exp (-\mathrm{i} \omega t)]$, and again $\phi$ satisfies the two-dimensional Helmholtz equation, where now $k$ is the positive solution to the dispersion relation $k \tanh k h=\omega^{2} / g$ and $g$ is the acceleration due to gravity. The appropriate boundary condition is then $\partial \phi / \partial n=0$.

Consider a periodic array of identical circles of radius $a$ the centres of which are located on the line $y=0$ in the $(x, y)$ plane, with the $j$ th scatterer centred at $(j, 0)$. Clearly the 


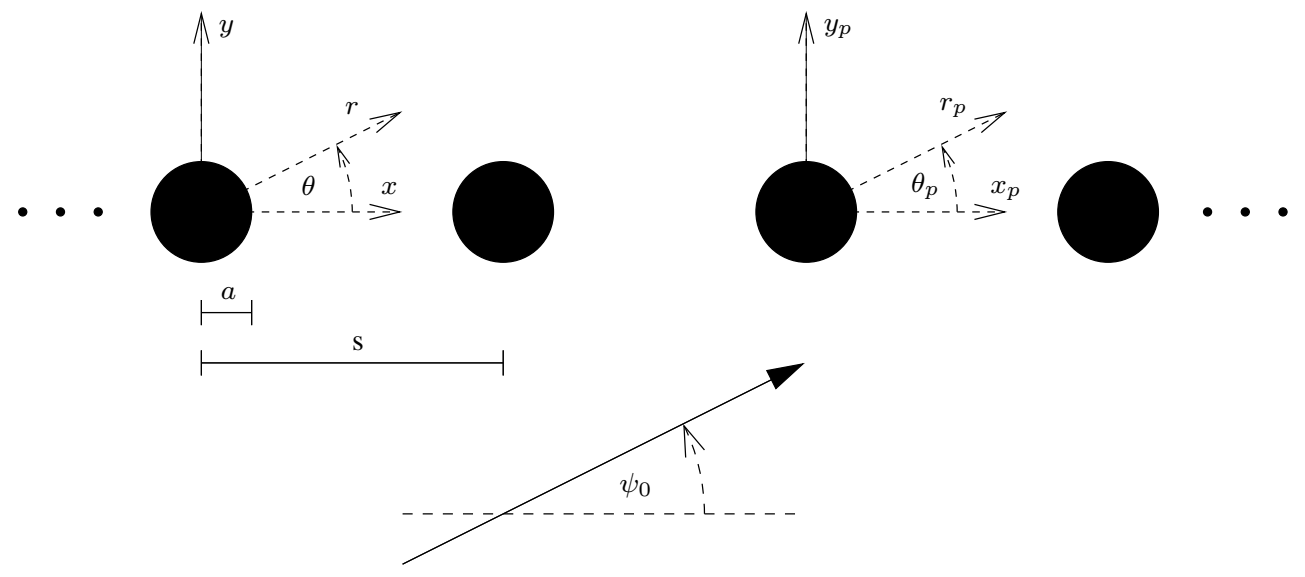

Figure 1: The infinite array, with scatterers centred at $(p, 0)$ for integer $p$, and with a plane wave incident at angle $\psi_{0}$.

radius of the scatterers must satisfy $0<a<0.5$. The plane wave

$$
\phi^{\mathrm{i}}=\mathrm{e}^{\mathrm{i} k\left(x \cos \psi_{0}+y \sin \psi_{0}\right)}
$$

is incident at angle $\psi_{0}$ upon the array as shown in figure 1 . The total field is expressed in the form

$$
\phi^{\mathrm{t}}=\phi^{\mathrm{i}}+\phi^{\mathrm{s}}
$$

where $\phi^{\mathrm{s}}$ is the scattered response from the array. Introducing shifted sets of polar coordinates $\left(r_{p}, \theta_{p}\right)$ with the origin positioned at the centre of scatterer $p$, the boundary condition on the surface of the scatterers requires that either

$$
\partial \phi^{\mathrm{t}} / \partial r_{p}=0 \quad \text { on } \quad r_{p}=a, \quad p \in \mathbb{Z}
$$

for the Neumann problem, or

$$
\phi^{\mathrm{t}}=0 \quad \text { on } \quad r_{p}=a, \quad p \in \mathbb{Z},
$$

for the Dirichlet problem. The scattered field can be represented in the form

$$
\phi^{\mathrm{s}}=\sum_{p=-\infty}^{\infty} \sum_{n=-\infty}^{\infty} B_{n}^{p} \mathrm{H}_{n}\left(k r_{p}\right) \mathrm{e}^{\mathrm{i} n \theta_{p}}
$$

where $\mathrm{H}_{n}(\cdot)$ is the $n$th order Hankel function of the first kind. An infinite system of equations for the unknown constants $B_{n}^{p}$ can be obtained by applying boundary conditions on the surface of the scatterers using Graf's addition theorem [9, eqn 9.1.79] as in Linton \& McIver [10]. Thus,

$$
B_{m}^{p}+Z_{m} \sum_{\substack{j=-\infty \\ j \neq p}}^{\infty} \sum_{n=-\infty}^{\infty} B_{n}^{j} X_{n-m}^{j p} \mathrm{H}_{n-m}(k|j-p|)=-Z_{m} \mathrm{i}^{m} \mathrm{e}^{\mathrm{i} k p \cos \psi_{0}} \mathrm{e}^{-\mathrm{i} m \psi_{0}}, \quad m, p \in \mathbb{Z},
$$


where

$$
X_{q}^{j p}= \begin{cases}(-1)^{q} & : j>p, \\ 1 & : j<p .\end{cases}
$$

For the Neumann condition (4), the coefficients $Z_{n}$ take the form

$$
Z_{n}=\mathrm{J}_{n}^{\prime}(k a) / \mathrm{H}_{n}^{\prime}(k a),
$$

whereas for the Dirichlet condition (5) we have

$$
Z_{n}=\mathrm{J}_{n}(k a) / \mathrm{H}_{n}(k a) .
$$

In either case, $Z_{-n}=Z_{n}$. For a finite array, the system of equations $(7)$ can be solved efficiently by truncation, since the spatial summation (in $j$ ) terminates, whereas the order summation (over $n$ ) converges very rapidly.

For the infinite array, we may seek a solution in which the only difference between the field at $x=x_{0}$ and that at $x=x_{0}+p$ is the phase factor $\mathrm{e}^{\mathrm{i} k p \cos \psi_{0}}$, so that

$$
B_{n}^{p}=\mathrm{e}^{\mathrm{i} k p \cos \psi_{0}} B_{n}^{0}=\mathrm{e}^{\mathrm{i} k p \cos \psi_{0}} B_{n},
$$

say. The infinite system (7) then reduces to

$$
B_{m}+Z_{m} \sum_{n=-\infty}^{\infty} B_{n} \sigma_{n-m}=-Z_{m} \mathrm{i}^{m} \mathrm{e}^{-\mathrm{i} m \psi_{0}}, \quad m \in \mathbb{Z},
$$

in which

$$
\sigma_{n}=\sum_{j=1}^{\infty}\left[\mathrm{e}^{-\mathrm{i} j k \cos \psi_{0}}+(-1)^{n} \mathrm{e}^{\mathrm{i} j k \cos \psi_{0}}\right] \mathrm{H}_{n}(j k) .
$$

The quantity $\sigma_{n}$ is a specific type of Schlömilch series often referred to as a lattice sum; its value can be computed efficiently using expressions given in $[11,12]$. Note that we ignore the possibility of Rayleigh-Bloch surface waves which propagate along the array, as their presence in the solution is precluded by the phase relationship (10). In any case, such waves typically exist when $k<\pi$ [13], whereas resonance requires that $k \geq \pi$, as we shall see.

Once the coefficients $B_{n}$ are obtained, the total field at any point can be evaluated using (6), though this procedure is computationally expensive due to the slow convergence of the spatial summation. A useful alternative representation can be obtained by observing that, close to cylinder $p$, the total field can be written as

$$
\phi^{\mathrm{t}}=\phi_{p}^{\mathrm{i}}+\mathrm{e}^{\mathrm{i} k p \cos \psi_{0}} \sum_{n=-\infty}^{\infty} B_{n} \mathrm{H}_{n}\left(k r_{p}\right) \mathrm{e}^{\mathrm{i} n \theta_{p}}
$$

where $\phi_{p}^{\mathrm{i}}$ is the total field incident on cylinder $p$ made up from the incident plane wave and the scattered waves from all the other cylinders. Since $\phi_{p}^{\mathrm{i}}$ is regular for $r_{p}<1$ it can be expanded in this region as

$$
\phi_{p}^{\mathrm{i}}=\sum_{n=-\infty}^{\infty} C_{n}^{p} \mathrm{~J}_{n}\left(k r_{p}\right) \mathrm{e}^{\mathrm{i} n \theta_{p}}
$$


for some set of coefficients $C_{n}^{p}$. The boundary condition (4) or (5) then shows that

$$
\phi^{\mathrm{t}}=\mathrm{e}^{\mathrm{i} k p \cos \psi_{0}} \sum_{n=-\infty}^{\infty} B_{n} \mathrm{e}^{\mathrm{i} n \theta_{p}}\left(\mathrm{H}_{n}\left(k r_{p}\right)-\frac{\mathrm{J}_{n}\left(k r_{p}\right)}{Z_{n}}\right), \quad r_{p}<1 .
$$

This form is particularly useful for computing the field near $y=0$.

We now express the scattered field as a sum of plane waves and evanescent modes. This is the most descriptive representation of the solution. Thus, we introduce a small damping factor by writing $k=k_{r}+\mathrm{i} \epsilon$, where $\epsilon>0, k_{r} \in \mathbb{R}$, and insert the integral representation [14]

$$
\mathrm{H}_{n}(k r) \mathrm{e}^{\mathrm{i} n \theta}=\frac{(-\mathrm{i})^{n+1}}{\pi} \int_{-\infty}^{\infty}\left[\frac{\alpha-\gamma(\alpha)}{k}\right]^{n \operatorname{sgn}(y)} \mathrm{e}^{-\gamma(\alpha)|y|+\mathrm{i} \alpha x} \frac{\mathrm{d} \alpha}{\gamma(\alpha)}
$$

into (6). Here $\gamma(\alpha)=\left(\alpha^{2}-k^{2}\right)^{1 / 2}$, with $\gamma(0)=-\mathrm{i} k$, and the damping factor moves the branch points off the real line. Note that when $|\alpha / k| \leq 1$ the term in square brackets is simply $\exp (\mathrm{i} \arccos (\alpha / k))$. Taking the limit $\epsilon \rightarrow 0$ determines the direction in which the integration contour should be indented in order to obtain the appropriate time-harmonic solution. In fact, the contour must be indented above the singularity at $\alpha=-k$ and below that at $\alpha=k$. For $n \neq 0$ this representation is valid everywhere except on $y=0$. For $n=0$ it is valid everywhere except at $x=y=0$.

The Poisson summation formula then yields

$$
\phi^{\mathrm{s}}=\sum_{j=-\infty}^{\infty} A_{j} \mathrm{e}^{\mathrm{i} k\left(x \cos \psi_{j}+|y| \sin \psi_{j}\right)},
$$

for $y \neq 0$, where the scattering angles $\psi_{j}$ are given by

$$
k \cos \psi_{j}=k \cos \psi_{0}+2 j \pi,
$$

and the amplitude coefficients by

$$
A_{j}=\frac{2}{k \sin \psi_{j}} \sum_{n=-\infty}^{\infty}(-\mathrm{i})^{n} B_{n} \mathrm{e}^{\mathrm{i} n \operatorname{sgn}(y) \psi_{j}} .
$$

Note that a finite number (at least one) of the quantities $\psi_{j}$ are real and satisfy $0 \leq \psi_{j} \leq \pi$ and these terms in (17) correspond to plane waves scattered off the array in the direction $\theta=\operatorname{sgn}(y) \psi_{j}$. For all $j$ we have

$$
\gamma\left(k \cos \psi_{j}\right)=-\mathrm{i} k \sin \psi_{j},
$$

and the terms for which $\left|\cos \psi_{j}\right|>1$ represent evanescent modes, which can be neglected for large $|y|$. At low frequencies, only a small number of plane waves are scattered by the array, and therefore (17) provides a great deal of insight into the solution.

Clearly the representation (17) breaks down if $\sin \psi_{p}=0$ for any $p$ because then the amplitude coefficient $A_{p}$ is undefined. Under these circumstances, one of the scattering angles is either 0 or $\pi$; so that one of the scattered plane waves propagates along the array. This is the resonant case that we analyse next, and which is the main subject of this article. There are two possibilities: either there is one value of $p$ for which $\sin \psi_{p}=0$ (single resonance) or there are two (double resonance). 


\section{Single resonance}

Single resonance occurs if $\sin \psi_{p}=0$ for some unique $p \in \mathbb{Z}$. Exploiting the symmetry of the problem, we restrict our attention to the case in which $\psi_{p}=0$. Note that, from (18), this can only happen if

$$
p=0 \quad \text { or } \quad 0<p \leq k / \pi .
$$

The latter case clearly requires $k \geq \pi$. The quantity $A_{p}$ cannot be obtained directly from (19), nor, in fact can $B_{n}$ be computed from (11) since the Schlömilch series (12) is now divergent. Nevertheless, all physical quantities remain finite, and can be determined as follows.

Expressions for the Schlömilch series in terms of the scattering angles are given in [12]. The expansion for $\sigma_{n}$ is of the form

$$
\sigma_{n}=\mu_{n}+\frac{2(-\mathrm{i})^{n}}{k} \sum_{j=-\infty}^{\infty} \frac{\mathrm{e}^{\mathrm{i} n \operatorname{sgn}(j) \psi_{j}}}{\sin \psi_{j}}
$$

where $\mu_{n}$ is always bounded and in which we use the convention that $\operatorname{sgn}(0)=+1$. Thus, the crucial step is to consider the Schlömilch series $\sigma_{n}$ and the coefficient $B_{n}$ as functions of the scattering angle $\psi_{p}$. The Schlömilch series $\sigma_{n}\left(\psi_{p}\right)$ then has a singularity at the point $\psi_{p}=0$, but the total residue obtained from the summation in (11) must be zero in order to yield a finite right-hand side. Hence, we write

$$
\sigma_{n}\left(\psi_{p}\right)=\hat{\sigma}_{n}\left(\psi_{p}\right)+2(-\mathrm{i})^{n} /\left(k \psi_{p}\right),
$$

where $\hat{\sigma}_{n}$ remains bounded as $\psi_{p} \rightarrow 0$ (and is easily computed) and then introduce the Taylor expansion

$$
\frac{2}{k} \sum_{n=-\infty}^{\infty}(-\mathrm{i})^{n} B_{n}\left(\psi_{p}\right)=a_{1} \psi_{p}+O\left(\psi_{p}^{2}\right) .
$$

After substituting (23) into (11), we evaluate at $\psi_{p}=0$ to obtain

$$
B_{m}(0)+Z_{m} \sum_{n=-\infty}^{\infty} B_{n}(0) \hat{\sigma}_{n-m}(0)=-Z_{m} \mathrm{i}^{m}\left[\mathrm{e}^{-\mathrm{i} m \psi_{0}}+a_{1}\right], \quad m \in \mathbb{Z} .
$$

The presence of the extra unknown $a_{1}$ necessitates the use of an additional equation to close the system; this is obtained by evaluating $(24)$ at $\psi_{p}=0$ :

$$
\sum_{n=-\infty}^{\infty}(-\mathrm{i})^{n} B_{n}(0)=0
$$

Together, (25) and (26) constitute an infinite system of equations for the unknowns $B_{n}(0)$ and $a_{1}$. Expanding $\exp \left(\operatorname{in} \operatorname{sgn}(y) \psi_{p}\right)$ in powers of $\psi_{p}$, and using (24) in (19), we can now take the limit $\psi_{p} \rightarrow 0$ to obtain

$$
A_{p}=a_{1}+\operatorname{sgn}(y) \frac{2 \mathrm{i}}{k} \sum_{n=-\infty}^{\infty} n(-\mathrm{i})^{n} B_{n}(0) .
$$


In the case $p=0$ (grazing incidence), the entire system can be solved by inspection; we find that $B_{n}(0)=0$ and $a_{1}=-1$. Thus in the limit $\psi_{0} \rightarrow 0$, the scattered response eliminates the incident wave, i.e.

$$
\phi^{\mathrm{s}} \sim-\mathrm{e}^{\mathrm{i} k x}
$$

and the array behaves like a simple Dirichlet boundary located on $y=0$. (Note that this is independent of the actual boundary conditions that are applied on the scatterers themselves.)

\section{Double resonance}

Double resonance occurs if $\psi_{p}=0$ and $\psi_{q}=\pi$ at the same incidence angle $\psi_{0}$. From equation (18), we see immediately that this is possible when

$$
k=(p-q) \pi .
$$

Note that $\psi_{p}=0$ can only happen if (21) is satisfied and then it follows immediately that $q \leq 0$. We can ignore the case $q=0$ as, by symmetry, this is equivalent to the case $p=0$. Therefore we consider $q<0$. When (29) holds we have the relationship

$$
k \cos \psi_{q}=k \cos \psi_{p}-2 k .
$$

Again considering the Schlömilch series as functions of $\psi_{p}$, we must determine the nature of the singularity located at the origin. As before, the term $j=p$ in (22) gives rise to a singularity of the form $2(-\mathrm{i})^{n} /\left(k \psi_{p}\right)$, but now we must also take the term $j=q$ into account. From equations (20) and (30), we have

$$
\sin \psi_{q}=\gamma\left(k \cos \psi_{p}-2 k\right)
$$

and hence there is a now branch point merged with the pole at the origin in the $\psi_{p}$ plane. By leaving undetermined the direction in which this point is to be approached, we shall show that this causes no significant difficulty. A straightforward calculation shows that if $\left|\psi_{p}\right|$ is small, then

$$
\psi_{q}=\pi \pm \mathrm{i} \psi_{p}+O\left(\psi_{p}^{3}\right)
$$

with the lower sign to be taken if $\psi_{p} \rightarrow 0$ through positive values and the upper sign corresponding to $\psi_{p} \rightarrow 0$ through purely imaginary values. This shows that if mode $p$ is cutting off then mode $q$ must be cutting on, and vice versa. We then find that

$$
\frac{\mathrm{e}^{-\mathrm{i} n \psi_{q}}}{\sin \psi_{q}}=\frac{ \pm \mathrm{i}(-1)^{n}}{\psi_{p}}+\mathrm{i} n(-1)^{n}+O\left(\psi_{p}\right) .
$$

Thus

$$
\sigma_{n}\left(\psi_{p}\right)=\hat{\sigma}_{n}\left(\psi_{p}\right)+\frac{2 \mathrm{i}^{n}}{k \psi_{p}}\left[(-1)^{n} \pm \mathrm{i}\right],
$$

where $\hat{\sigma}_{n}\left(\psi_{p}\right)$ again remains bounded as $\psi_{p} \rightarrow 0$ and is easily computed. 
The summation in (11) must yield a finite result whichever sign is taken and the coefficients $B_{n}(0)$ that result must be the same. Hence we can introduce the additional Taylor expansion

$$
\frac{2 \mathrm{i}}{k} \sum_{n=-\infty}^{\infty} \mathrm{i}^{n} B_{n}\left(\psi_{p}\right)= \pm b_{1} \psi_{p}+O\left(\psi_{p}^{2}\right)
$$

alongside (24), the sign corresponding to that chosen in (31). Substituting (33) into (11), and evaluating at $\psi_{p}=0$ we find that

$$
B_{m}(0)+Z_{m} \sum_{n=-\infty}^{\infty} B_{n}(0) \hat{\sigma}_{n-m}(0)=-Z_{m} \mathrm{i}^{m}\left[\mathrm{e}^{-\mathrm{i} m \psi_{0}}+a_{1}+(-1)^{m} b_{1}\right], \quad m \in \mathbb{Z} .
$$

The system is then closed by evaluating (24) and (34) at $\psi_{p}=0$. Finally, we find that $A_{p}$ is unchanged from the case of single resonance, i.e. it is given by (27), whilst for $A_{q}$, we deduce from (31) and (32) that

$$
A_{q}=b_{1}-\operatorname{sgn}(y) \frac{2 \mathrm{i}}{k} \sum_{n=-\infty}^{\infty} n \mathrm{i}^{n} B_{n}(0) .
$$

The entire system can be solved by inspection for $\psi_{0}=0(p=0$ and $q=-k / \pi)$. We find that $B_{n}(0)=0$, with $a_{1}=-1, b_{1}=0$ and once again the scattered field satisfies

$$
\phi^{\mathrm{s}} \sim-\mathrm{e}^{\mathrm{i} k x} .
$$

\section{$5 \quad$ Numerical results and discussion}

The results in this section were computed by truncating the order summation at $n=15$, i.e. taking 31 unknowns per scatterer. For small values of $a$, the incident field dominates both above and below the array, and we have chosen to take $a=0.25$ in order to give greater relative significance to other effects, and to resonance in particular. In general, more interesting phenomena are found when the Neumann (sound hard) boundary condition is applied on the surface of the scatterers; therefore most of the results presented in this section are for this case.

If we define transmission and reflection coefficients for the array via

$$
T_{j}=\delta_{j 0}+A_{j}
$$

for $y>0$, and

$$
R_{j}=A_{j}
$$

for $y<0$, then conservation of energy requires that

$$
\sin \psi_{0}=\sum_{j} \sin \psi_{j}\left(\left|R_{j}\right|^{2}+\left|T_{j}\right|^{2}\right)
$$

where the summation in $j$ ranges over those values that correspond to propagating modes, and excludes those that are associated with evanescent waves (for a derivation see, e.g., 


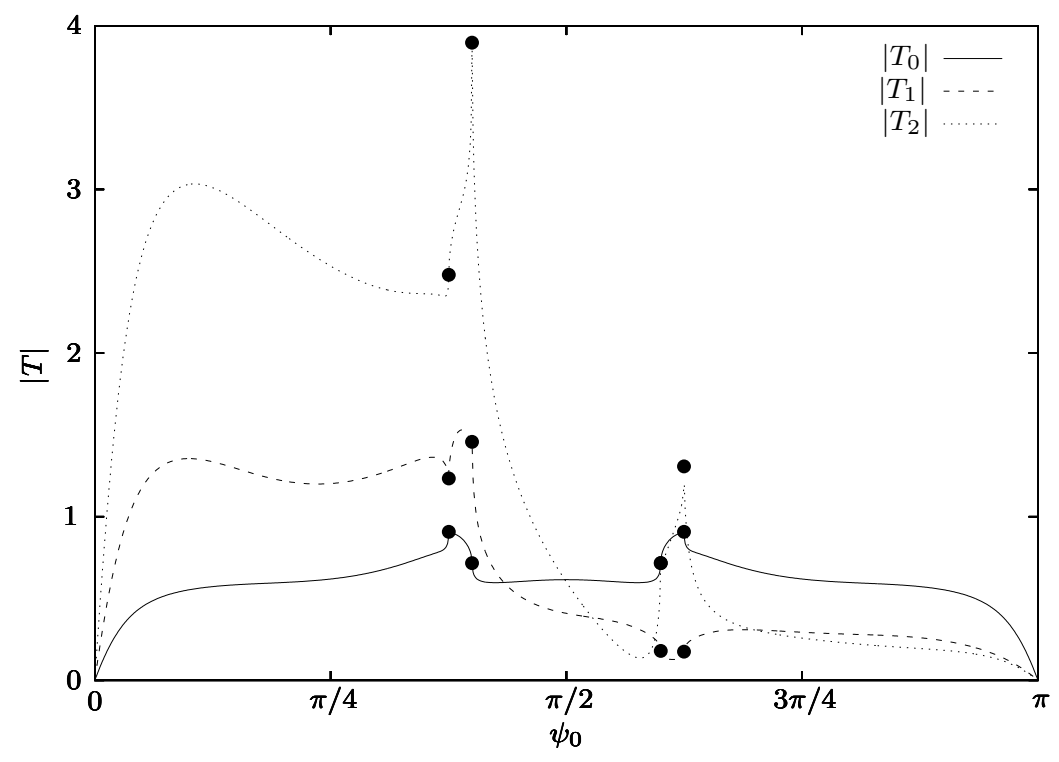

Figure 2: Mode amplitudes for $a=0.25, k \approx 9.1$. The black disks $(\bullet)$ indicate the amplitude when one of the modes is resonant.

[15]). This is a necessary, though not sufficient, condition for the correctness of our results. In particular, note that resonant modes do not contribute to the right-hand side of (40). Thus the results were also validated by checking that the modal form of the solution satisfies the boundary condition on the surface of the scatterers. Symmetry applies between modes $j$ and $-j$, thus

$$
\left.T_{j}\right|_{\psi_{0}}=\left.T_{-j}\right|_{\pi-\psi_{0}}
$$

and likewise for $R_{j}$.

Figure 2 shows three mode amplitudes above the array in the Neumann case, with varying $\psi_{0}$. The wavenumber is chosen so that mode 1 is right resonant at $\psi_{0}=0.4 \pi$, hence from (18), $k \approx 9.1$. At this value, the resonance is clearly the dominant effect, since, although $\left|T_{2}\right|$ is large, modes with $j \geq 2$ are evanescent in this case. Similarly, modes with $j \leq-2$ are also evanescent, and the amplitude of mode -1 can be deduced from that of mode 1 at $\psi_{0}=0.6 \pi$. The quantity $T_{0}$ refers to the transmission of the incident field through the array; here the curve is symmetric about $\psi_{0}=\pi / 2$. Equation (11) was used for the continuous curves, with one thousand data points on a uniform mesh. Sharp changes in the gradients for all of the modes occur close to resonances; there are four in this case. Thus, a second right resonance occurs for mode 2 at $\psi_{0} \approx 0.62 \pi$, and there are corresponding left resonances at $\psi_{0}=0.6 \pi$ (mode -1$)$ and $\psi_{0} \approx 0.38 \pi$ (mode -2 ). Calculations at the resonances using $(25)$ are marked with black disks. It is difficult to accurately compute the mode amplitudes by setting $\psi_{0}$ close to the critical angle; this is particularly evident in the plot for $T_{2}$. In order to make a comparison between the results at resonance for a periodic array, and those for the same parameters but for a large, finite array, equation (7) was solved for 101 scatterers. 


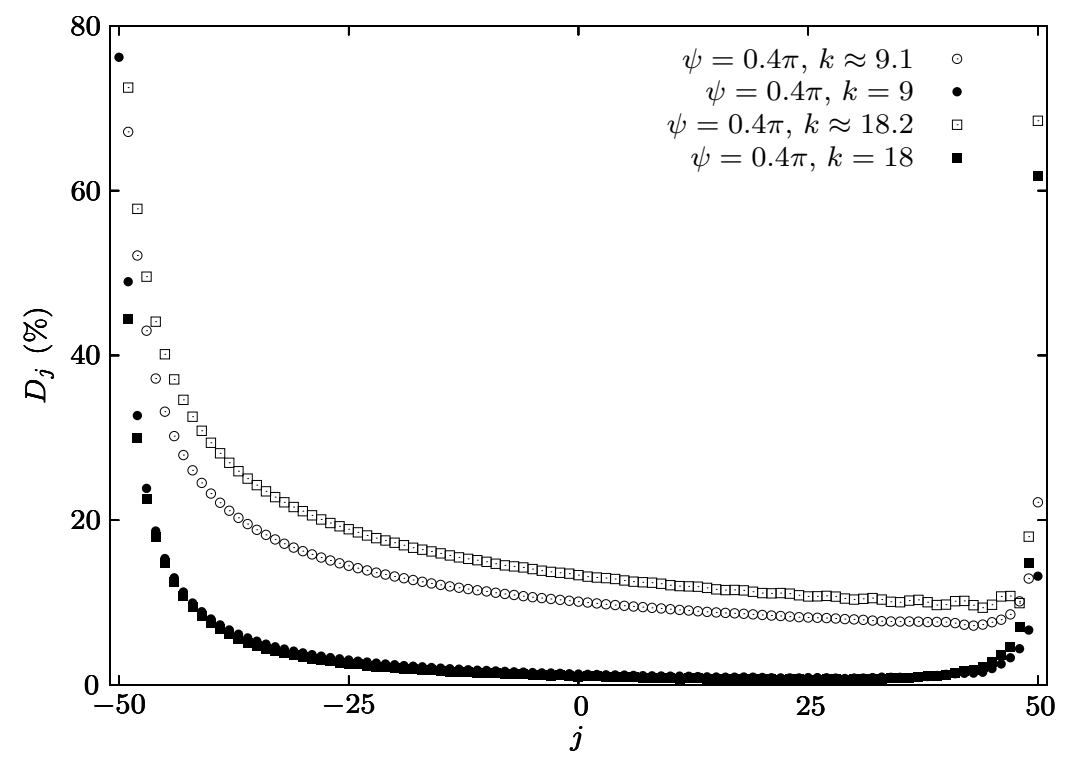

Figure 3: Percentage difference (see equation (42)) between the periodic solution and that for a 101 scatterer array. All results are for the Neumann case.

We then define the percentage difference on cylinder $j$ as

$$
D_{j}=100 \times \frac{\sum_{n=-\infty}^{\infty}\left|B_{n}^{j}-\mathrm{e}^{\mathrm{i} k j \cos \psi_{0}} B_{n}\right|}{\sum_{n=-\infty}^{\infty}\left|B_{n}^{j}\right|} .
$$

Values of $D_{j}$ for two resonant and two near resonant cases are shown in figure 3 . In each case, the difference is greatest close to the ends, as expected. The end effects are much stronger in the resonant cases, and also increase slightly with frequency. The left end has much greater influence than the right; this situation is reversed if we take $\psi_{0}>\pi / 2$. A comprehensive investigation of end effects in finite arrays will form the basis of a future paper.

A contour plot of the $\operatorname{Re}\left[\phi^{\mathrm{t}}\right]$ with $\psi_{0}=0.4 \pi$ and mode 1 resonant is shown in figure 4 , and its finite array counterpart in figure 5 . The position along the array was chosen as the location of best agreement from figure 3 and is close to the right end of the finite array. The results are indeed very similar. The field tends to be slightly stronger in the periodic case, whilst the finite array solution exhibits more variation as the observer moves away from the array. Note that the dominance of the resonant mode above the array predicted by figure 2 is clearly evident in figure 4. Amplitudes of the propagating modes in figure 4 and all subsequent contour plots are shown to two significant figures in table 1. As before, $\left|T_{0}\right|$ represents the strength of the incident field above the array. Obviously, the incident field is also present (with unit amplitude) beneath the array. When a single mode has significantly greater amplitude than all of the others, a clear pattern is evident, such as that in figure 4; more interference is visible when this is not the case, as we shall see.

A contour plot of $\operatorname{Re}\left[\phi^{t}\right]$ in a near resonant state, with $\psi_{0}=0.4 \pi$ and $k=9$ is shown in figure 6. Note the marked difference between this and the field in figure 4, despite the relatively small change in wavenumber. Close to the array, there is some evidence of resonance, however mode 1 is now evanescent, and therefore this decays rapidly as $|y|$ is 


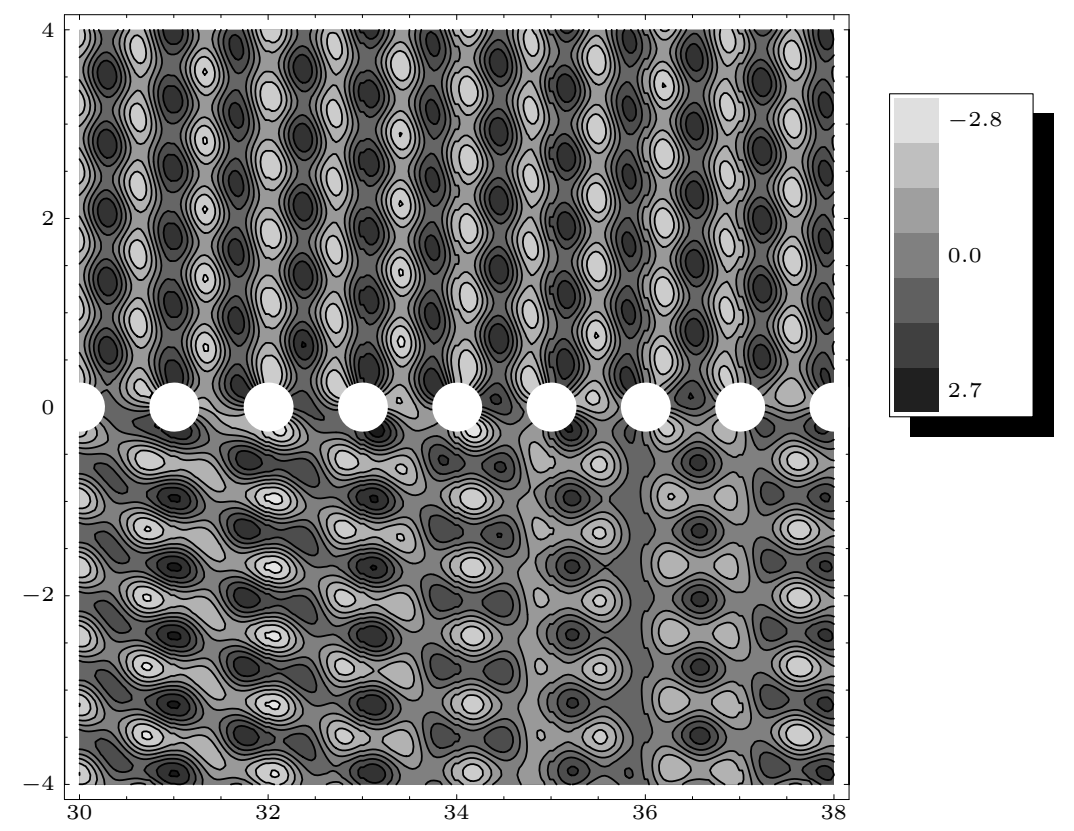

Figure 4: Contour plot of $\operatorname{Re}\left[\phi^{\mathrm{t}}\right]$ for the infinite array in the Neumann case, with $\psi_{0}=0.4 \pi$ and $k \approx 9.1$. Mode 1 is resonant.

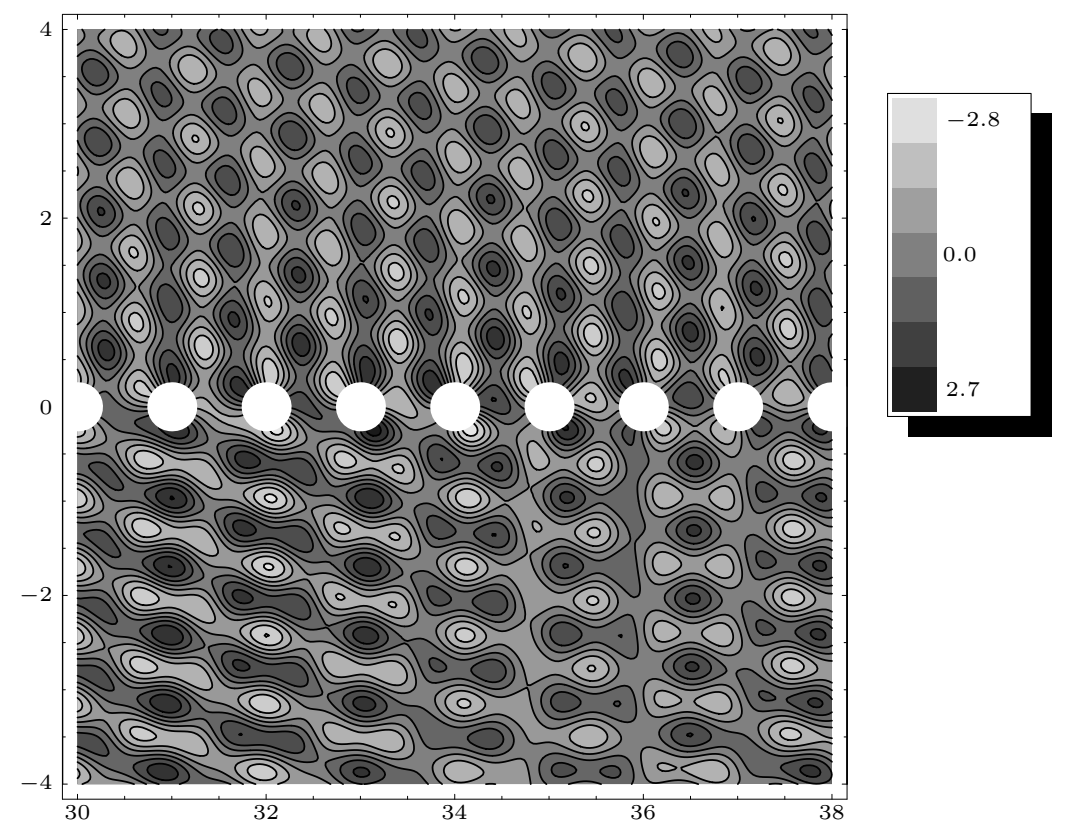

Figure 5: Contour plot of $\operatorname{Re}\left[\phi^{\mathrm{t}}\right]$ in the interior of a 101 scatterer array in the Neumann case, with $\psi_{0}=0.4 \pi$ and $k \approx 9.1$. 


\begin{tabular}{|c|c|c|c|c|c|c|c|}
\hline & $\begin{array}{r}\text { Fig. } \\
k \\
\psi_{0}\end{array}$ & $\begin{array}{c}4 \\
\approx 9.1 \\
0.4 \pi\end{array}$ & $\begin{array}{c}6 \\
9.0 \\
0.4 \pi\end{array}$ & $\begin{array}{c}\quad 7 \\
\approx 18.2 \\
0.4 \pi\end{array}$ & $\begin{array}{c}8 \\
\approx 10.4 \\
0.8 \pi\end{array}$ & $\begin{array}{c}9 \\
\approx 10.4 \\
0.8 \pi\end{array}$ & $\begin{aligned} & 10 \\
& 3 \pi \\
\approx & 0.62 \pi\end{aligned}$ \\
\hline \multirow{7}{*}{$y>0$} & $\left|T_{-3}\right|$ & - & - & 0.57 & - & - & - \\
\hline & $\left|T_{-2}\right|$ & - & - & 0.042 & - & - & - \\
\hline & $\left|T_{-1}\right|$ & 0.18 & 0.15 & 0.41 & - & - & $2.2 \leftarrow$ \\
\hline & $\left|T_{0}\right|$ & 0.71 & 0.78 & 0.24 & 0.08 & 0.015 & 0.79 \\
\hline & $\left|T_{1}\right|$ & $1.5 \rightarrow$ & - & 0.40 & 0.36 & 0.11 & 0.084 \\
\hline & $\left|T_{2}\right|$ & - & - & $0.75 \rightarrow$ & 0.20 & 0.19 & $0.78 \rightarrow$ \\
\hline & $\left|T_{3}\right|$ & - & - & - & $1.1 \rightarrow$ & $0.12 \rightarrow$ & - \\
\hline \multirow{7}{*}{$\mathbf{y}<\mathbf{0}$} & $\left|R_{-3}\right|$ & - & - & 0.31 & - & - & - \\
\hline & $\left|R_{-2}\right|$ & - & - & 0.16 & - & - & - \\
\hline & $\left|R_{-1}\right|$ & 0.34 & 0.34 & 0.20 & - & - & $1.4 \leftarrow$ \\
\hline & $\left|R_{0}\right|$ & 0.59 & 0.50 & 0.48 & 0.62 & 0.58 & 0.60 \\
\hline & $\left|R_{1}\right|$ & $0.68 \rightarrow$ & - & 0.22 & 0.31 & 0.39 & 0.064 \\
\hline & $\left|R_{2}\right|$ & - & - & $1.9 \rightarrow$ & 0.34 & $0.12 \rightarrow$ & $1.8 \rightarrow$ \\
\hline & $\left|R_{3}\right|$ & - & - & - & $0.96 \rightarrow$ & - & - \\
\hline
\end{tabular}

Table 1: Amplitudes (to two significant figures) for the propagating modes in figures 4, 7, 8, $9 \& 10$. All of the values shown here are for the Neumann case, except those corresponding to figure 9 . Resonant modes are denoted by arrows. 


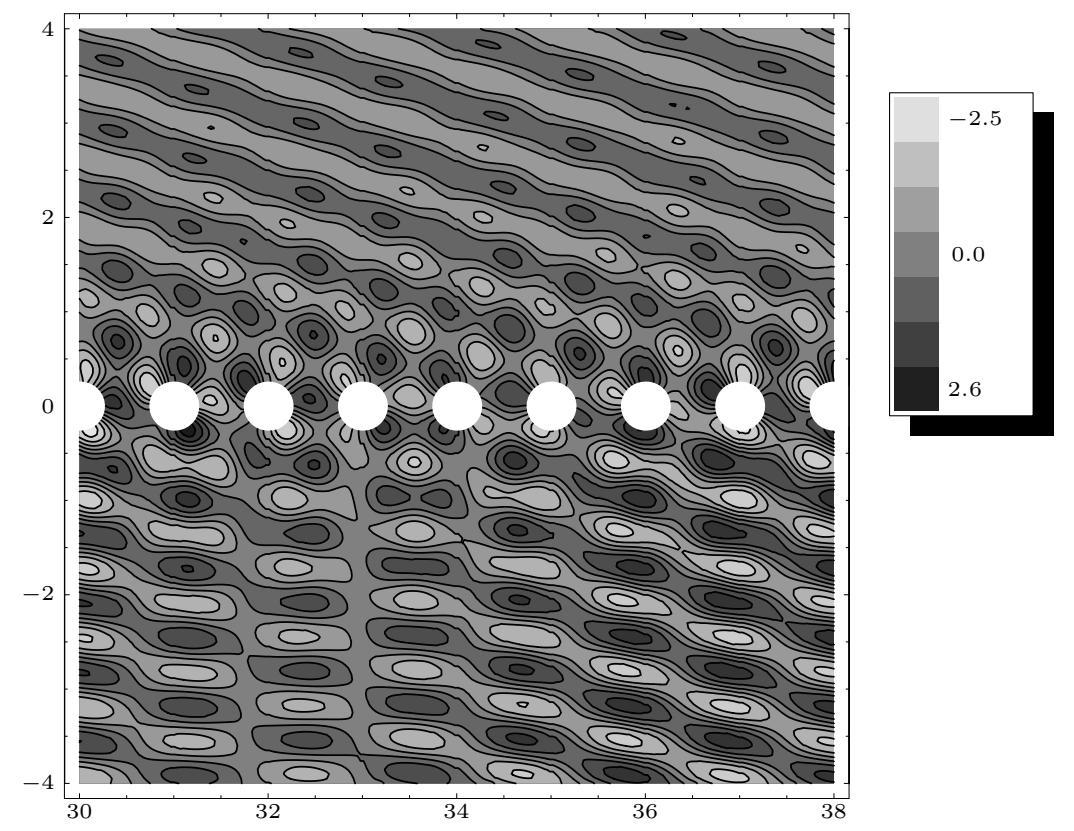

Figure 6: Contour plot of $\operatorname{Re}\left[\phi^{\mathrm{t}}\right]$ for the infinite array, using Neumann boundary conditions, with $\psi_{0}=0.4 \pi$ and $k=9.0$.

increased. Transmission is clearly the strongest effect for $y>1$. A higher order resonance is shown in figure 7. Here, the frequency has been doubled, so that mode 2 is now resonant at $\psi_{0}=0.4 \pi$. As predicted by the values given in table 1 , the resonance is the strongest below the array. Above the array, however, the resonance is much weaker, allowing modes -3 and \pm 1 to create a great deal of interference. The angle of incidence has been increased to $0.8 \pi$ in figure 8 . Nevertheless, a strong right resonance (mode 3 ) is clearly evident above the array. The transmission of the incident wave is particularly weak in this case. Beneath the array, the incident, reflected and resonant fields are of comparable amplitudes, leading to interference.

A contour plot for the same parameters (i.e. $\psi_{0}=0.8 \pi$ and mode 3 resonant, but with Dirichlet boundary conditions now applied on the surface of the scatterers is shown in 9 . The transmission coefficients, and in particular that of the incident wave, are small, relative even to those for figure 8 . Thus, although the resonance is much less pronounced than in the Neumann case, it is still visible in the region above the array. An instance of double resonance is shown in figure 10 . Here, $k=3 \pi$, and the angle of incidence is chosen so that modes -1 and 2 are resonant, thus $\psi_{0} \approx 0.62 \pi$. The resonances are clearly the dominant effects both above and below the array.

\section{Acknowledgements}

IT is supported by EPSRC under grant EP/C510941/1. 


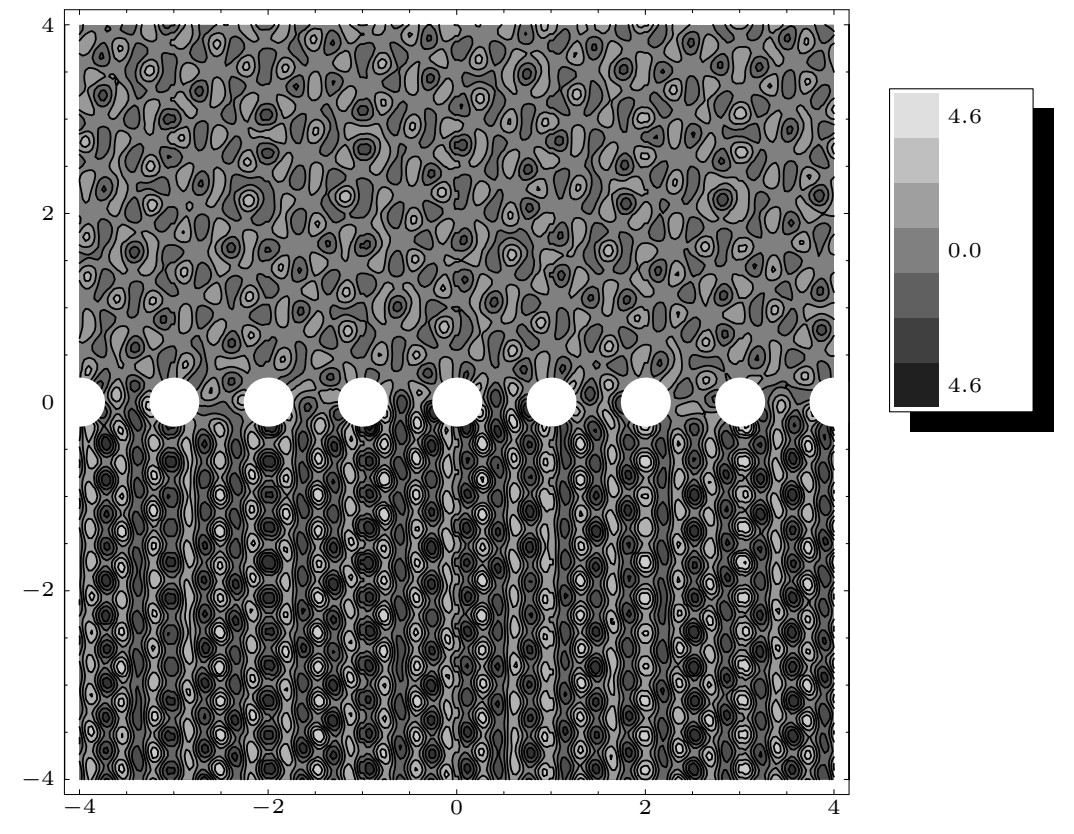

Figure 7: Contour plot of $\operatorname{Re}\left[\phi^{\mathrm{t}}\right]$ for the infinite array in the Neumann case, with $\psi_{0}=0.4 \pi$ and $k \approx 18.2$. Mode 2 is resonant.

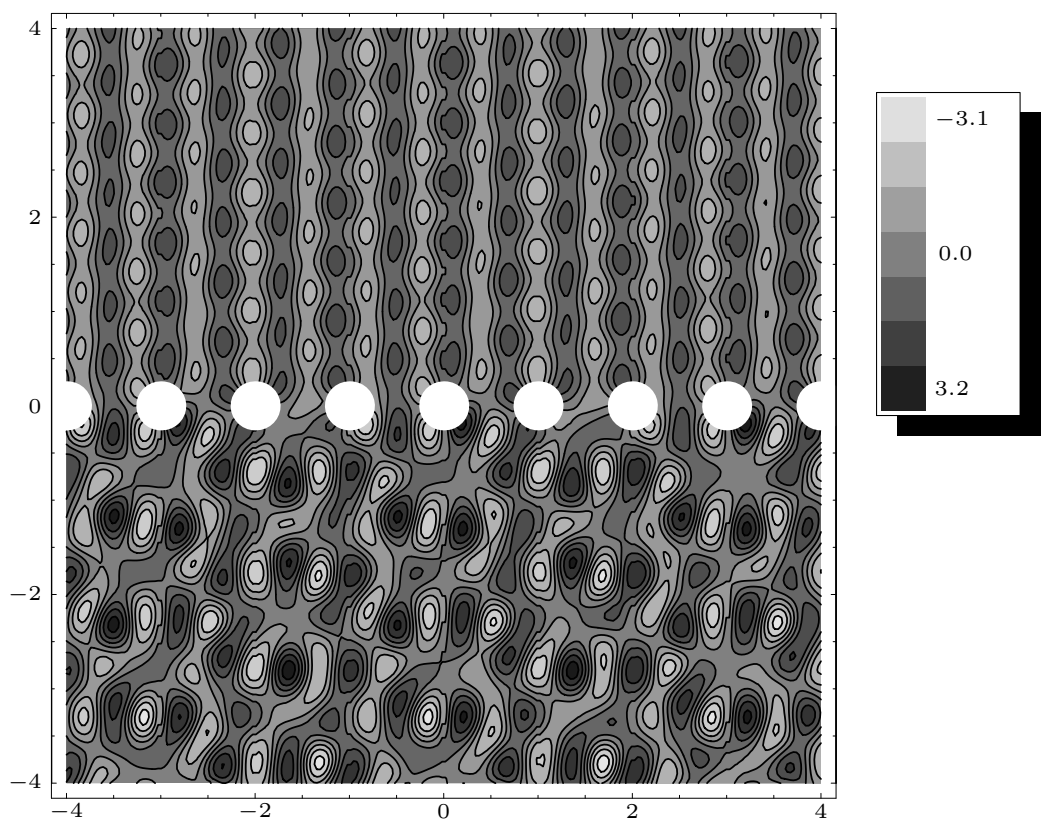

Figure 8: Contour plot of $\operatorname{Re}\left[\phi^{t}\right]$ for the infinite array in the Neumann case, with $\psi_{0}=0.8 \pi$ and $k \approx 10.4$. Mode 3 is resonant. 


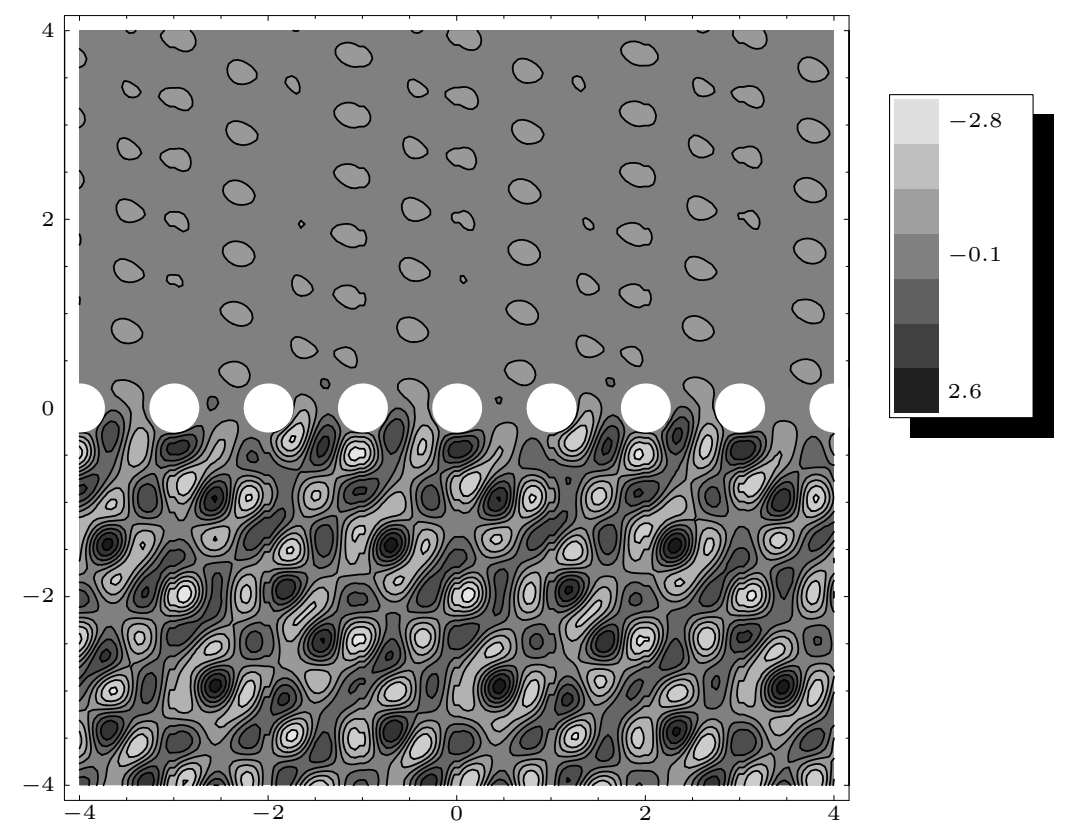

Figure 9: Contour plot of $\operatorname{Re}\left[\phi^{t}\right]$ for the infinite array, using Dirichlet boundary conditions, with $\psi_{0}=0.8 \pi$ and $k \approx 10.4$. Mode 3 is resonant.

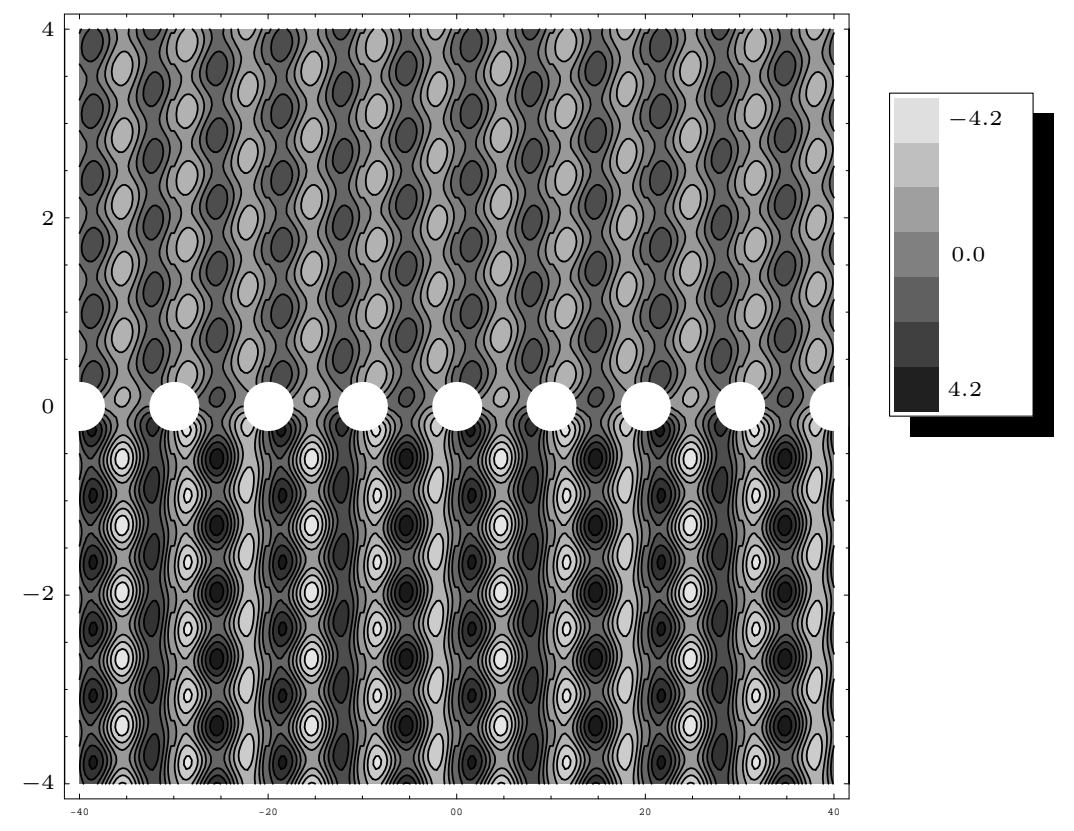

Figure 10: Contour plot of $\operatorname{Re}\left[\phi^{\mathrm{t}}\right]$ for the infinite array, using Neumann boundary conditions, with $\psi_{0} \approx 0.61 \pi$ and $k=3 \pi$. Modes 2 and -1 are resonant. 


\section{References}

[1] R. W. Wood. On a remarkable case of uneven distribution of light in a diffraction grating spectrum. Phil. Mag., 4:396-408, 1902.

[2] J. W. Rayleigh. On the dynamical theory of gratings. Proc. Roy. Soc. Lond., A, 79:399416, 1907.

[3] A. Hessel and A. A. Oliner. A new theory of Wood's anomalies on optical gratings. Applied Optics, 4(10):1275-1297, 1965.

[4] V. Twersky. On scattering of waves by the infinite grating of circular cylinders. IRE Trans. on Antennas and Propagation, 10:737-765, 1962.

[5] R. C. McPhedran and M. D. Waterworth. A theoretical demonstration of properties of grating anomalies (S polarization). Optica Acta, 19(11):877-892, 1972.

[6] R. C. McPhedran and M. D. Waterworth. Properties of diffraction grating anomalies. Optica Acta, 20(7):533-547, 1973.

[7] Wenhao Zhu, Michael R. Stinson, and Gilles A. Daigle. Scattering from impedance gratings and surface wave formation. J. Acoust. Soc. Am., 111(5):1996-2012, 2002.

[8] I. Thompson and C. M. Linton. On the excitation of a closely spaced array by a line source. Submitted.

[9] M. Abramowitz and I. A. Stegun. Handbook of Mathematical Functions. Dover Publications, New York, 1965.

[10] C. M. Linton and P. McIver. Handbook of Mathematical Techniques for Wave/Structure Interactions. Chapman \& Hall/CRC, Boca Raton, 2001.

[11] V. Twersky. Elementary function representation of Schlömilch series. Arch. Rational Mech. Anal., 8:323-332, 1961.

[12] C. M. Linton. Schlömilch series that arise in diffraction theory and their efficient computation. J. Phys. A, 39:3325-3339, 2006.

[13] R. Porter and D. V. Evans. Rayleigh-Bloch surface waves along periodic gratings and their connection with trapped modes in waveguides. J. Fluid Mech., 386:233-258, 1999.

[14] C. M. Linton and D. V. Evans. The radiation and scattering of surface waves by a vertical circular cylinder in a channel. Phil. Trans. R. Soc. Lond., A, 338:325-357, 1992.

[15] J. D. Achenbach, Y.-C. Lu, and M. Kitahara. 3-D reflection and transmission of sound by an array of rods. J. Sound Vib., 125(3):463-476, 1988. 